Corrigendum

\title{
Corrigendum to "Anxiety and Anger Symptoms in Hwabyung Patients Improved More following 4 Weeks of the Emotional Freedom Technique Program Compared to the Progressive Muscle Relaxation Program: A Randomized Controlled Trial"
}

\author{
Jin Woo Suh, Sun Yong Chung, Sang Young Kim, Jung Hwan Lee, and Jong Woo Kim \\ Department of Korean Neuropsychiatry, Graduate School, Kyung Hee University, 26 Kyungheedae-ro, Dongdaemun-gu, \\ Seoul 02447, Republic of Korea \\ Correspondence should be addressed to Jong Woo Kim; aromaqi@khu.ac.kr \\ Received 15 September 2016; Accepted 20 September 2016 \\ Copyright (C) 2016 Jin Woo Suh et al. This is an open access article distributed under the Creative Commons Attribution License, \\ which permits unrestricted use, distribution, and reproduction in any medium, provided the original work is properly cited.
}

In the article titled "Anxiety and Anger Symptoms in Hwabyung Patients Improved More following 4 Weeks of the Emotional Freedom Technique Program Compared to the Progressive Muscle Relaxation Program: A Randomized Controlled Trial" [1], the authors' affiliations were incorrectly listed. The correct affiliation is shown above.

\section{References}

[1] J. W. Suh, S. Y. Chung, S. Y. Kim, J. H. Lee, and J. W. Kim, "Anxiety and anger symptoms in hwabyung patients improved more following 4 weeks of the emotional freedom technique program compared to the progressive muscle relaxation program: a randomized controlled trial," Evidence-based Complementary and Alternative Medicine, vol. 2015, Article ID 203612, 9 pages, 2015. 


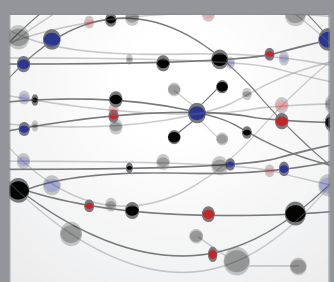

The Scientific World Journal
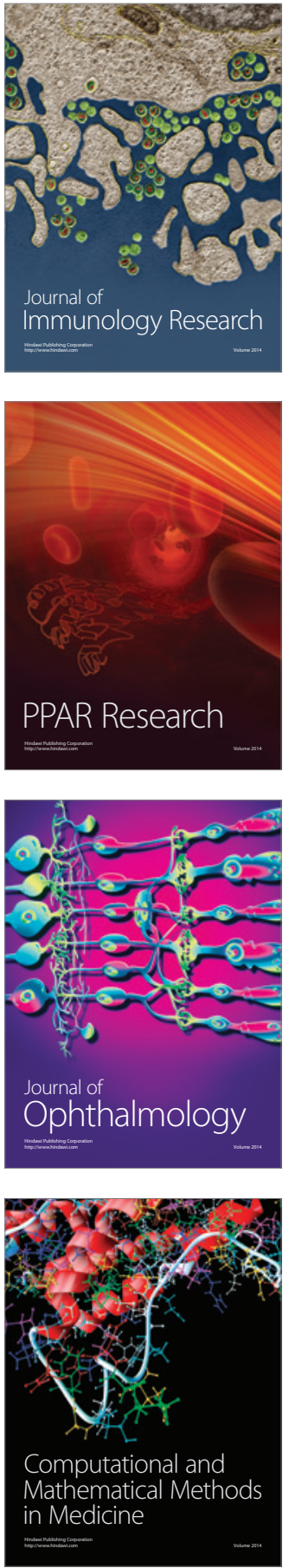

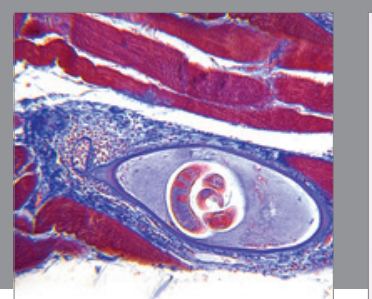

Gastroenterology Research and Practice

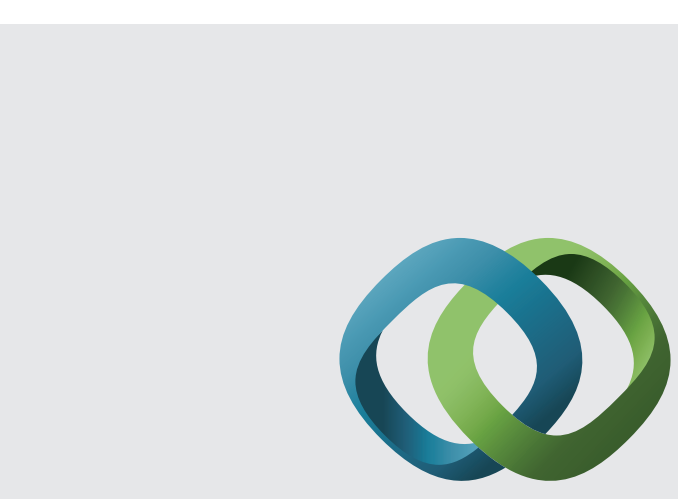

\section{Hindawi}

Submit your manuscripts at

http://www.hindawi.com
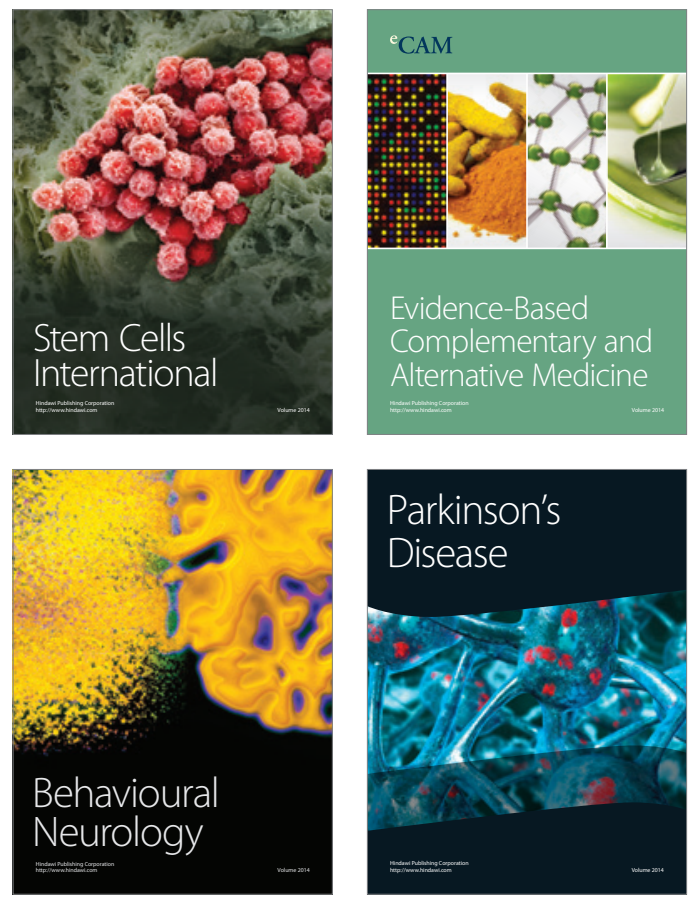
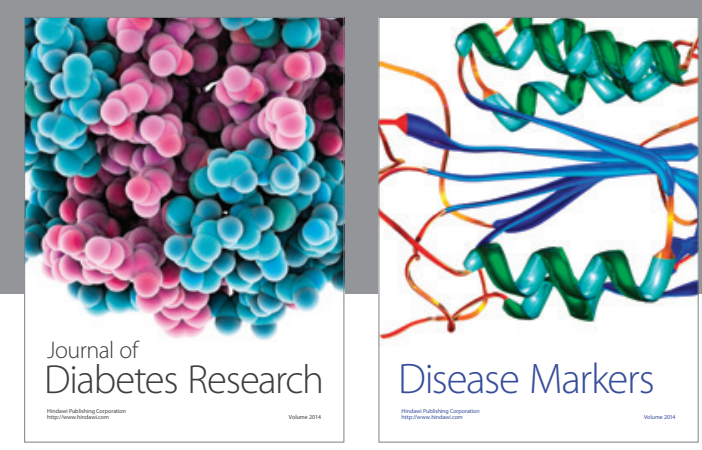

Disease Markers
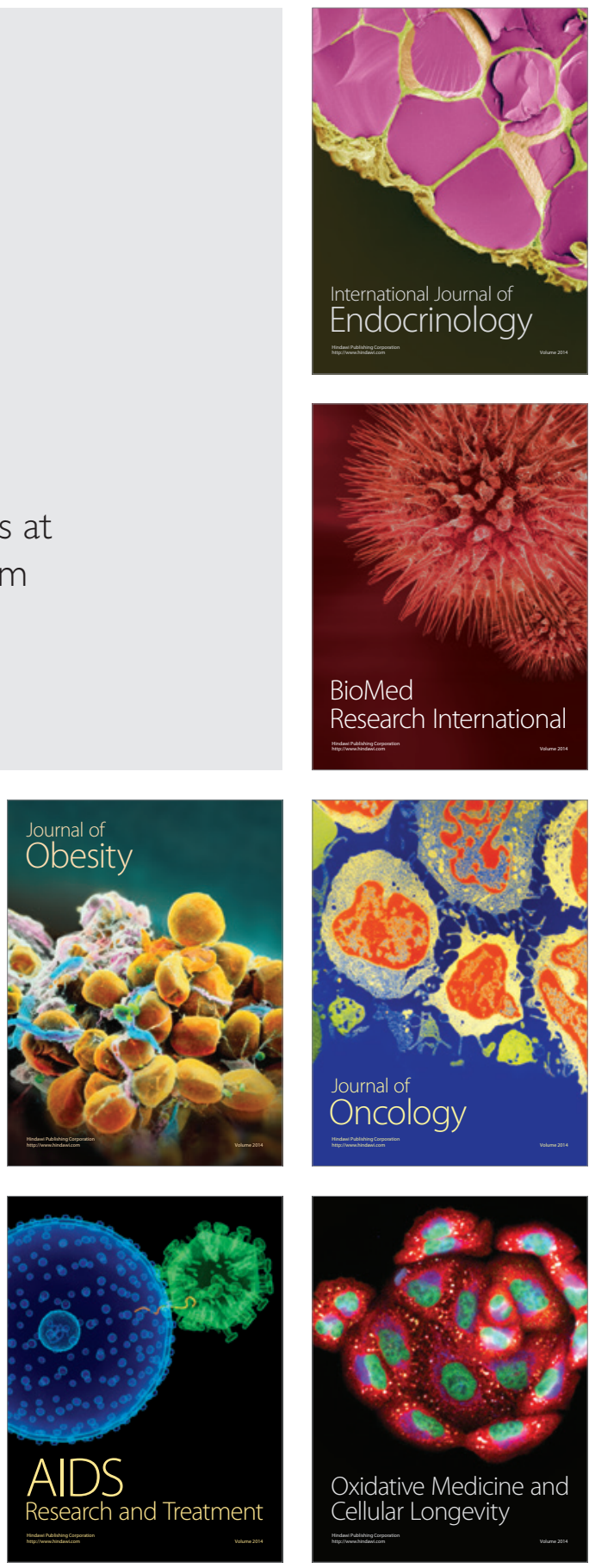